\title{
Duplex synthetic aperture imaging with tissue motion compensation
}

\author{
Gammelmark, Kim; Jensen, Jørgen Arendt
}

Published in:

2003 IEEE International Ultrasonics Symposium Proceedings

Link to article, DOI:

10.1109/ULTSYM.2003.1293207

Publication date:

2003

Document Version

Publisher's PDF, also known as Version of record

Link back to DTU Orbit

Citation (APA):

Gammelmark, K., \& Jensen, J. A. (2003). Duplex synthetic aperture imaging with tissue motion compensation. In 2003 IEEE International Ultrasonics Symposium Proceedings (Vol. 2, pp. 1569-1573). IEEE. I E E E International Ultrasonics Symposium. Proceedings https://doi.org/10.1109/ULTSYM.2003.1293207

\section{General rights}

Copyright and moral rights for the publications made accessible in the public portal are retained by the authors and/or other copyright owners and it is a condition of accessing publications that users recognise and abide by the legal requirements associated with these rights.

- Users may download and print one copy of any publication from the public portal for the purpose of private study or research.

- You may not further distribute the material or use it for any profit-making activity or commercial gain

- You may freely distribute the URL identifying the publication in the public portal 


\title{
Duplex Synthetic Aperture Imaging with Tissue Motion Compensation
}

\author{
Kim Løkke Gammelmark and Jørgen Arendt Jensen* \\ Center for Fast Ultrasound Imaging, Ørsted•DTU, Build. 348, \\ Technical University of Denmark, 2800 Kgs. Lyngby, Denmark
}

\begin{abstract}
This paper investigates a method for tissue motion estimation and compensation in synthetic transmit aperture imaging. The approach finds the tissue velocity and the direction of the motion at every tissue region by crosscorrelating high resolution lines beamformed along multiple directions at each image points. Compensation is applied in the beamformer by tracking the image points using the velocity and angle estimates from the closest estimation point. Simulation results using Field II show nearly perfect motion compensation with no appreciable difference in contrast resolution after compensation. Phantom measurements show similar performance with differences in contrast resolution of $29 \%$ and $0.61 \%$ before and after compensation, respectively.
\end{abstract}

\section{INTRODUCTION}

Synthetic transmit aperture (STA) imaging has been investigated by several research groups over the last decade, e.g. $[1,2]$. The results presented have in general shown that STA imaging has the potential to improve image quality beyond that obtained by the traditional imaging techniques like linear/phased array imaging. So far, however, in-vivo implementation of STA imaging has generally been limited by two main problems: 1) Low signal-to-noise ratio (SNR), and 2) Susceptibility to tissue motion.

The low SNR arises due to the classic application of a single transmit element. This limits the penetration of the ultrasound beam in the presence of attenuation, which is a general property of biological tissue, and thus minimizes the clinical utility of the approach. In recent papers $[1,3]$ it has however been shown, that the loss in SNR can be overcome by replacing the single transmit element with a subaperture defocused to emulate a high power spherical wave, and at the same time replace the traditional short excitation with a long linear frequency modulated (FM) waveform [4]. This approach, denoted TMS imaging ${ }^{1}$, increases the SNR by several $\mathrm{dB}$ compared to e.g. linear array imaging, which makes in-vivo imaging possible. Thus, the short penetration depth is

\footnotetext{
*This work is sponsored by grant 9700883 and 9700563 from the Danish Science Foundation, by Ph.D. grant 11-3160-55173 from the Technical University of Denmark, and by B-K Medical A/S, Gentofte, Denmark.

${ }^{1}$ Temporally encoded $\underline{\text { Multi-element }} \underline{\text { Synthetic transmit aperture (TMS) }}$ imaging
}

no longer an issue, which requires attention.

STA imaging relies on the coherent summation of sequentially acquired and beam formed low resolution images. Thus, STA imaging is susceptible to tissue motion, because this will cause the low resolution images to be incoherently summed, resulting in an unfocused image. This susceptibility is more pronounced for STA imaging compared to traditional imaging, because the latter techniques build up the image line by line. Tissue motion therefore distorts the structures in the traditional images rather than degrading the spatial and contrast resolutions. If tissue motion is severe, successful STA imaging requires motion compensation. This is especially the case, if several emissions are used for each high resolution image.

This paper presents a method for tissue motion compensation in STA imaging based on cross-correlation of high resolution image lines beamformed along the direction of the motion. The method and acquisition strategy is described in Section II, and simulations with Field II used to theoretically investigate the approach are presented in Section III. Phantom measurement results obtained using the RASMUS research scanner are presented in Section IV, and the paper is concluded in Section V.

\section{Motion COMPENSATION}

Coherent summation of the low resolution images beamformed after each emission in STA imaging is necessary in order to fully utilize the advantages of the method. If these images are not phase aligned, incoherent summation will occur which produces an unfocused, smeared high resolution image. This affects the SNR, spatial resolution, and contrast resolution. Such phase misalignment is produced by target motion, where the tissue moves between each emission. Thus, it is necessary to estimate this motion and compensate for it in order to maintain image quality.

Different methods for motion estimation and compensation have been studied for synthetic aperture ultrasound imaging previously, e.g. [5, 6]. As reported in these papers, the dominant factor in image degradation is axial motion due to the significantly higher spatial frequency in this direction. Lateral motion will however also have a significant impact on 


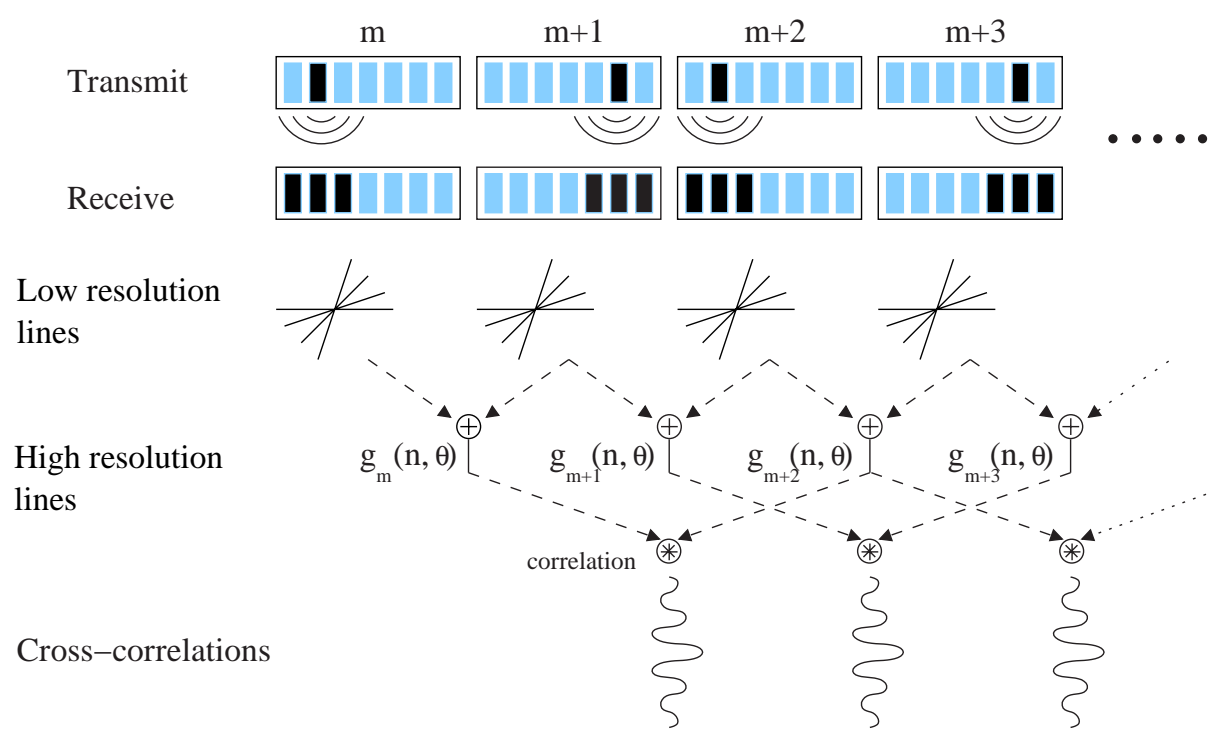

Figure 1: Illustration of the tissue velocity estimation approach.

the image quality. This is especially the case, if many emissions are used, because the motion artifacts build up. Therefore, two dimensional motion compensation is necessary.

Generally, motion estimation methods are based on crosscorrelation of reference signals to find the shift in position or phase of the tissue. The degree of success of such methods depends on the correlation between the reference signals, which again depends on the degree of common spatial frequencies used to generate the reference signals. One method to obtain highly correlated reference signals is to use high resolution images. These will be completely correlated, if they are acquired in exactly the same way. This property has recently been used to generate synthetic aperture flow images in $[7,8]$, where the blood flow velocity is estimated by crosscorrelating high resolution lines acquired using TMS imaging with few emissions and beamformed along the flow direction.

The motion estimation and compensation method presented here is based on the synthetic aperture flow method mentioned above. The approach utilizes the high correlation between the high resolution images and the fact that for a given emission any set of image points within the interrogated region can be created using STA beamforming [7]. The method is illustrated in Fig. 1 using two emissions. For a given image point at which tissue motion is to be estimated, a set of low resolution lines along selected directions are beam formed for each emission. For each set of consecutive emissions, here $[m, m+1],[m+1, m+2],[m+2, m+3]$, etc., the corresponding lines for each angle $\theta$ are then summed to form high resolution lines $g_{m}(n, \theta), g_{m+1}(n, \theta), g_{m+2}(n, \theta)$, etc., where $n$ is the sample number. Assuming a constant tissue velocity during the acquisition, the distortion in $g_{m}(n, \theta)$ and $g_{m+2}(n, \theta)$ will be the same and the only difference is the shift in position of the scatterers. The correlation for the different angles will be highest in the direction of the motion. By crosscorrelating $g_{m}(n, \theta)$ and $g_{m+2}(n, \theta)$ for all angles, the direction of the motion is therefore found at the angle for which the normalized correlation coefficient is maximum. For this angle the shift in position can be found and thus the velocity.

The shift in position between high resolution lines $g_{m+1}(n, \theta)$ and $g_{m+3}(n, \theta)$ will be equal to that between $g_{m}(n, \theta)$ and $g_{m+2}(n, \theta)$, if the tissue velocity is assumed constant. Thus, the peak in the cross-correlation functions will be located at the same lag, and they can be averaged to improve the velocity estimate. This averaging can be applied for as long as the velocity can be assumed constant.

In general, $M$ emissions, denoted flow mode emissions or F-mode emissions, are used, and the emission sequence is repeated $N_{\text {seq }}$ times. The $i$ 'th high resolution line is created by

$$
g_{i}\left(n, \theta, \vec{r}_{p}\right)=\sum_{m=1}^{M} s_{m+i}\left(n, \theta, \vec{r}_{p}\right),
$$

where $n, n=1,2, \ldots, N$, is the sample number along the line, $\theta$ is the angular direction of the line, and $\vec{r}_{p}$ denotes the location of the point being investigated (see Fig. 2). The signal $s_{m}\left(n, \theta, \vec{r}_{p}\right)$ is the low resolution line for emission $m$, and $i=1,2, \ldots, M\left(N_{\text {seq }}-1\right)$. The number of samples $N$ in the directional lines and the sampling interval $\delta r$ are determined by several parameters, e.g. the spatial frequency at the given angle $\theta$, and the maximum tissue velocity.

The normalized cross-correlation is obtained by

$$
\rho_{i, i+M}\left(k, \theta, \vec{r}_{p}\right)=\frac{E\left\{g_{i}\left(n, \theta, \vec{r}_{p}\right) g_{i+M}\left(n+k, \theta, \vec{r}_{p}\right)\right\}}{\sqrt{\sigma_{i}^{2} \sigma_{i+M}^{2}}},
$$




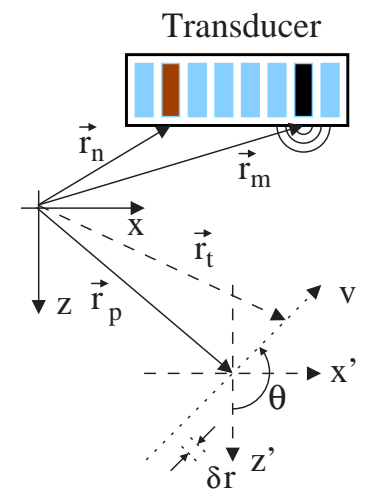

Figure 2: Definition of geometric variables.

where $k$ is the lag, and $\sigma_{i}^{2}$ and $\sigma_{i+M}^{2}$ are the variances of $g_{i}\left(n, \theta, \vec{r}_{p}\right)$ and $g_{i+M}\left(n, \theta, \vec{r}_{p}\right)$, respectively. Using the average cross-correlation function

$$
\bar{\rho}\left(k, \theta, \vec{r}_{p}\right)=\frac{1}{M\left(N_{\text {seq }}-1\right)} \sum_{i=1}^{M\left(N_{\text {seq }}-1\right)} \rho_{i, i+M}\left(k, \theta, \vec{r}_{p}\right)
$$

the direction of the motion is found at the angle $\hat{\theta}\left(\vec{r}_{p}\right)$ for which $\bar{\rho}\left(k, \theta, \vec{r}_{p}\right)$ is maximum as explained previously. The velocity magnitude $v_{m}\left(\vec{r}_{p}\right)$ of the motion is then calculated by

$$
\hat{v}\left(\vec{r}_{p}\right)=\frac{k_{\max }\left(\hat{\theta}\left(\vec{r}_{p}\right)\right) \delta r f_{p r f}}{M},
$$

where $k_{\max }\left(\hat{\theta}, \vec{r}_{p}\right)$ is lag of the cross-correlation peak, and $f_{p r f}$ is the pulse repetition frequency.

To obtain continuous B-mode and flow imaging, the TMS imaging emissions and the F-mode emissions are interleaved such that every second emission is an F-mode emission. Hereby, the motion can be estimated continuously. The velocity and angle estimates are used to compensate the low resolution B-mode images by recalculating the location of the image points to beamform. With reference to Fig. 2, the high resolution $\mathrm{B}$-mode image is created by

$$
S\left(\vec{r}_{p}\right)=\sum_{m=1}^{N_{x m t}} w_{m}\left(\vec{r}_{p}\right) \sum_{n=1}^{N_{r c v}} w_{n}\left(\vec{r}_{p}\right) g_{m, n}\left(\frac{d_{m, n}\left(\vec{r}_{t}(m)\right)}{c}\right),
$$

where $w_{n}\left(\vec{r}_{p}\right)$ is the apodization values for receive element $n$, $w_{m}(\vec{r})$ is the apodization value for transmit element $m, c$ is the sound speed, and $N_{x m t}$ and $N_{r c v}$ are the number of emissions and receive channels, respectively. The total distance $d_{m, n}\left(\vec{r}_{t}(m)\right)$ from transmission center $m$ to the tracked image point and back to receive element $n$ is given by

$$
d_{m, n}\left(\vec{r}_{t}(m)\right)=\left|\vec{r}_{t}(m)-\vec{r}_{m}\right|+\left|\vec{r}_{n}-\vec{r}_{t}(m)\right|,
$$

where

$$
\vec{r}_{t}(m)=[\sin \hat{\theta}, 0, \cos \hat{\theta}] \cdot \frac{2 \hat{v}}{f_{p r f}}(m-1)
$$

Note, that this approach can also be used for continuous Bmode and blood flow TMS imaging.

\section{SIMULATIONS}

To investigate the method described in the previous section theoretically, a simulation using Field II has been performed $[9,10]$. A commercial 128 element $7 \mathrm{MHz}$ linear array transducer with element spacing on the order of a wavelength $(\lambda)$ was used. Each emission is performed using a 33 element subaperture and with a $20 \mu$ s linear FM signal as excitation waveform. The linear FM signal and the corresponding compression filter have both been amplitude weighted to reduce the near and distant temporal sidelobes $[4,3]$. Each acquisition sequence consists of $96 \mathrm{~B}$-mode emissions and 12 motion sequences each with 8 F-mode emissions. This results in a total of 192 emissions in each acquisition and 89 crosscorrelation estimates. The B-mode and F-mode emissions are interleaved with ratio 1-to-1 as explained above. For every Bmode emission 64 receive elements spaced by $2 \lambda$ were used (i.e. every second transducer element). For the F-mode emissions the 64 element receive aperture was centered around the transmit subaperture.

A tissue mimicking phantom containing an anechoic cyst with a diameter of $4 \mathrm{~mm}$ centered on-axis and $25 \mathrm{~mm}$ away from the transducer was used as target. Uniform tissue motion was simulated by changing the position of the scatterers between each emission event. The velocity of the motion was $0.15 \mathrm{~m} / \mathrm{s}$, and the scatterers were moved at an angle $\theta=110^{\circ}$ relative to the $z$-axis, see Fig. 2 .

The velocity magnitude and angle were estimated for every $2 \mathrm{~mm}$ in both the lateral and axial direction. The relative bias and standard deviation (STD) of the velocity estimate obtained were $-4.40 \%$ and $0.85 \%$, respectively, while the relative bias and STD of the estimated angle were 1.08\% and $0.20 \%$, respectively. The resulting images are shown in Fig. 3. Clearly the motion completely destroys the image quality in the uncompensated image, while the image quality has been fully recovered after motion compensation.

The degradation in contrast resolution $C R_{r e l}$ in percent relative to the reference phantom is calculated by

$$
C R_{r e l}=\frac{C R_{r e f}-C R_{o b j}}{C R_{r e f}} \cdot 100,
$$

where $C R_{r e f}$ is the contrast resolution of the reference phantom, and $C R_{o b j}$ is the contrast resolution of the object being analyzed. The contrast resolution $C R$ is calculated by [3]

$$
C R=1-\frac{I_{C}}{I_{S}},
$$

where $I_{C}$ is the intensity inside the cyst, and $I_{S}$ is the intensity of the speckle. Using these equations, the contrast resolution before compensation is degraded by $48 \%$, while no appreciable difference is present after motion compensation. 

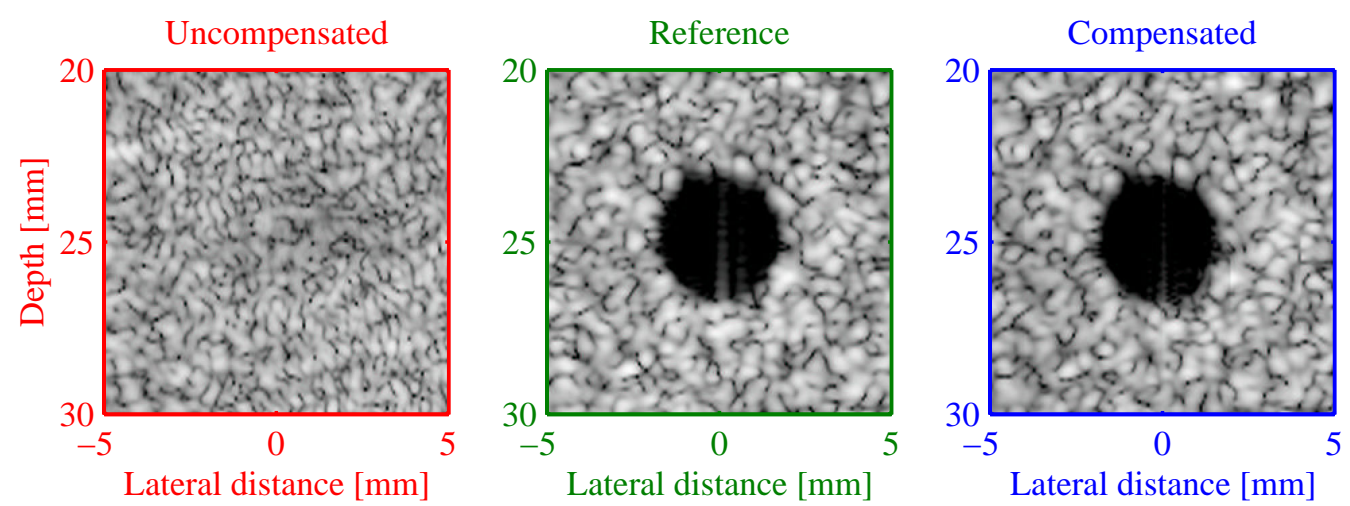

Figure 3: Image results of tissue motion simulation. Left: Uncompensated, Middle: Reference (stationary phantom). Right: Compensated. The dynamic range is $40 \mathrm{~dB}$.
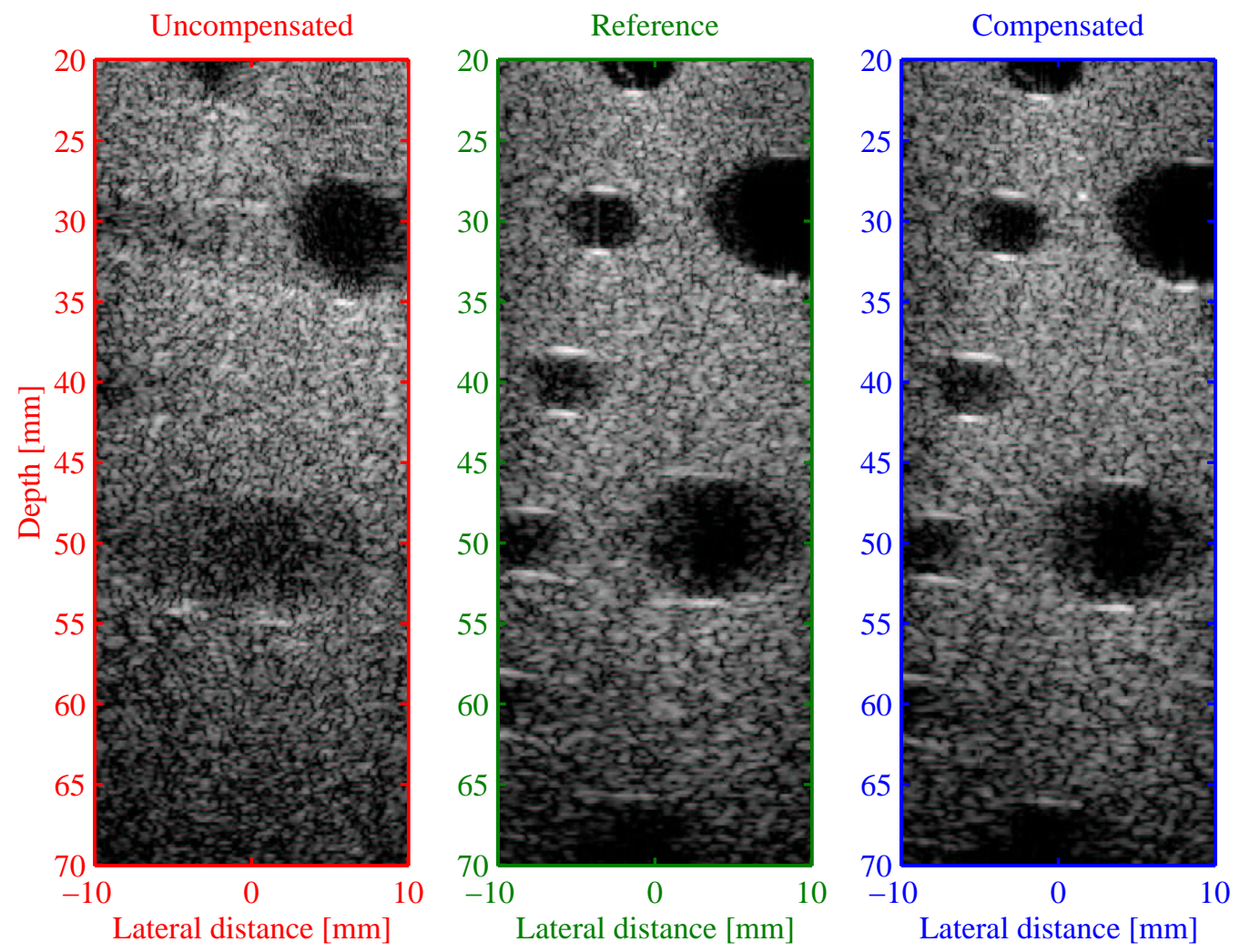

Figure 4: Left: Uncompensated, Middle: Reference (stationary phantom). Right: Compensated. The dynamic range is 50 dB.

\section{MEASUREMENTS}

Measurements were performed using the RASMUS research scanner developed at our center [11]. This system has
128 individually programmable transmitters capable of sending arbitrary coded waveforms with a precision of 12 bits at $40 \mathrm{MHz}$. Sixty-four receive channels are sampled simultaneously with a resolution of 12 bits and $40 \mathrm{MHz}$, and the 2-to-1 
multiplexing in the system enables acquisition of 128 channels in real-time over two transmissions.

An anechoic cyst phantom containing water filled cylinders with diameters of $4 \mathrm{~mm}$ and $8 \mathrm{~mm}$ was scanned. The applied acquisition and processing strategies were identical to those used in the simulation described in the previous section to enable direct comparison of the results. Uniform scatterer motion was emulated by moving the transducer between each emission using a high precision translation stage. The velocity and direction of the imposed motion was $0.106 \mathrm{~m} / \mathrm{s}$ and $-68.2^{\circ}$, respectively.

The tissue velocity and motion direction was estimated for every $2 \mathrm{~mm}$ in both the lateral and axial direction, and the angular separation between the directionally beam formed lines was $1^{\circ}$.

The relative bias and STD of the velocity estimate obtained were $-7.23 \%$ and $15.84 \%$, respectively, while the relative bias and STD of the estimated angle were $1.11 \%$ and $4.65 \%$, respectively. These numbers are higher than those obtained in the simulation, because they contain the false estimates from inside the cysts. These can be avoided by using a proper discriminator between noise and tissue. Neglecting the false estimates from inside the cysts, the relative bias and STD of the estimated velocity becomes $-3.24 \%$ and $5.63 \%$, while the angle estimates change to $-0.14 \%$ and $1.81 \%$.

The uncompensated, compensated, and reference B-mode images are shown in Fig. 4. As seen, a significant improvement in image quality has been obtained although the variance of the motion estimates is relatively high. The difference in contrast before and after compensation is $29 \%$ and $0.61 \%$, respectively, for the cyst located at depth $40 \mathrm{~mm}$.

\section{CONCLUSion}

A new method for tissue motion compensation was presented. The method estimated the velocity and direction of the tissue motion at every tissue region using crosscorrelation of high resolution lines beam formed along multiple direction. The direction of the motion is determined by the angle for which the correlation is maximized, and the velocity is found using conventional cross-correlation techniques. Compensation is performed using the velocity and angle estimates by recalculating the location of the image points, when beamforming the compensated low resolution images.

The simulation results showed nearly perfect motion compensation using the method. The difference in contrast resolution before and after compensation was $48 \%$ and approximately $0 \%$, respectively. The measurement results showed, that successful motion compensation can be obtained using the method in a noisy environment with only a $0.61 \%$ difference in contrast resolution after compensation.

\section{REFERENCES}

[1] M. Karaman, P. C. Li, and M. O’Donnell, "Synthetic aperture imaging for small scale systems," IEEE Trans. Ultrason., Ferroelec., Freq. Contr., vol. 42, pp. 429442, 1995.

[2] G. R. Lockwood, J. R. Talman, and S. S. Brunke, "Real-time 3-D ultrasound imaging using sparse synthetic aperture beamforming," IEEE Trans. Ultrason., Ferroelec., Freq. Contr., vol. 45, pp. 980-988, 1998.

[3] K. L. Gammelmark and J. A. Jensen, "Multielement synthetic transmit aperture imaging using temporal encoding," IEEE Trans. Med. Imag., vol. 22, no. 4, pp. 552-563, 2003.

[4] T. X. Misaridis and J. A. Jensen, "An effective coded excitation scheme based on a predistorted FM signal and an optimized digital filter," in Proc. IEEE Ultrason. Symp., 1999, vol. 2, pp. 1589-1593.

[5] G. E. Trahey and L. F. Nock, "Synthetic receive aperture imaging with phase correction for motion and for tissue inhomogenities - part II: effects of and correction for motion," IEEE Trans. Ultrason., Ferroelec., Freq. Contr., vol. 39, pp. 496-501, 1992.

[6] M. Karaman, H. Ş. Bilge, and M. O’Donnell, "Adaptive multi-element synthetic aperture imaging with motion and phase aberation correction," IEEE Trans. Ultrason., Ferroelec., Freq. Contr., vol. 42, pp. 1077-1087, 1998.

[7] S. I. Nikolov and J. A. Jensen, "Velocity estimation using synthetic aperture imaging," in Proc. IEEE Ultrason. Symp., 2001, pp. 1409-1412.

[8] J. A. Jensen and S. I. Nikolov, "Transverse flow imaging using synthetic aperture directional beamforming," in Proc. IEEE Ultrason. Symp., 2002, pp. 1488-1492.

[9] J. A. Jensen and N. B. Svendsen, "Calculation of pressure fields from arbitrarily shaped, apodized, and excited ultrasound transducers," IEEE Trans. Ultrason., Ferroelec., Freq. Contr., vol. 39, pp. 262-267, 1992.

[10] J. A. Jensen, "Field: A program for simulating ultrasound systems," Med. Biol. Eng. Comp., vol. 10th Nordic-Baltic Conference on Biomedical Imaging, Vol. 4, Supplement 1, Part 1, pp. 351-353, 1996 b.

[11] J. A. Jensen, O. Holm, L. J. Jensen, H. Bendsen, H. M. Pedersen, K. Salomonsen, J. Hansen, and S. Nikolov, "Experimental ultrasound system for real-time synthetic imaging," in Proc. IEEE Ultrason. Symp., 1999, vol. 2, pp. 1595-1599. 